\title{
Measurement of the cosmic ray Moon shadow with the ANTARES detector.
}

\author{
Matteo Sanguineti ${ }^{1,2, a}$ and Tommaso Chiarusi $i^{3, b}$ on behalf of ANTARES collboration \\ ${ }^{1}$ INFN - Sezione di Genova, Via Dodecaneso 33, 16146 Genova, Italy \\ ${ }^{2}$ Dipartimento di Fisica dell'Universita di Genova, Via Dodecaneso 33, 16146 Genova, Italy \\ ${ }^{3}$ INFN - Sezione di Bologna, Viale Berti-Pichat 6/2, 40127 Bologna, Italy
}

\begin{abstract}
The ANTARES detector is the largest neutrino telescope currently in operation in Mediterranean sea. One of the main goals of the ANTARES telescope is the search for point-like neutrino sources, so both the pointing accuracy and the angular resolution of the detector need a proper direct estimation. One possibility to evaluate the pointing performance of the detector is to analyse the shadow of the Moon, i.e. the deficit in the atmospheric muon flux in the direction of the Moon induced by absorption of cosmic rays. The ANTARES data taken between 2007 and 2016 shows a Moon shadow evidence of $3.5 \sigma$ significance. This is the first measurement of the ANTARES angular resolution and absolute pointing for atmospheric muons using a celestial calibration source. The presented results confirm the good pointing performance of the detector as well as the predicted angular resolution.
\end{abstract}

\section{Introduction}

The study of the Universe using cosmic neutrino as messanger opened a new era of astrophysics. Neutrinos are chargeless, weakly interacting particles; they can cross radiation fields or dense matter without being absorbed for cosmological distance. Neutrino are a complementary probe with respect to other messengers such as multi-wavelength electromagnetic radiation, charged cosmic rays and gravitational waves allowing thus the observation of the interior of the astrophysical sources and the far Universe. The ANTARES detector (Astronomy with a Neutrino Telescope and Abyss environmental RESearch) [1] is the largest neutrino telescope currently operating in sea water. The pointing accuracy and the angular resolution of the neutrino telescopes are critically important for the detection of point-like sources and a proper way to evaluate these performances is needed. Figures of merit depend on the relative delays among the optical sensors, but also on the instantaneous shape and absolute orientation of the detector lines.

In this measurement the Moon absorption of the cosmic rays is exploited to evidence a deficit in the event density of the atmospheric muon flux corresponding to the direction of the Moon disk in the ANTARES detector. The detection of this deficit - the so-called Moon shadow - and its position in the sky provides the measurements of the detector angular resolution for atmospheric down-going

\footnotetext{
a e-mail: matteo.sanguineti@ge.infn.it

be-mail: tommaso.chiarusi@bo.infn.it
} 
muons and the detector absolute pointing capability. The energy threshold for muons detectable at the depth of the ANTARES telescope is about $500 \mathrm{GeV}$ when they are at the sea surface level, most of them with energy above $1 \mathrm{TeV}$. Primaries which are progenitors of such highly energetic muons are practically not affected by the Earth geomagnetic field. This assumption of large rigidity holds also for the secondary muons detected by the ANTARES detector, thus they can be exploited in the study of the Moon shadow without introducing any bias.

\section{Moon shadow analysis optimization}

The optimization of the selection criteria applied to the reconstructed muon tracks has been performed using a dedicated Monte Carlo (MC) simulation with the MUPAGE code [2], it includes both single and bundle muon events. The Monte Carlo comprises the generation of Cherenkov light emitted by the muon tracks, its propagation up to the detector, the optical background caused by bioluminescence and the radioactive isotopes and the detector response. Finally the hits detected by the telescope are used to reconstruct the atmospheric muon tracks with a robust track fitting procedure based on a maximisation likelihood method [3].

The Moon shadow effect is simulated rejecting the muons generated within the Moon disk, having an angular radius $R_{\text {Moon }}=0.26^{\circ}$. In this analysis two different Monte Carlo simulation sets were performed: one considering the shadowing effect of the Moon $\left(H_{1}\right.$ hypothesis $)$ and the other without this effect $\left(H_{0}\right.$ hypothesis). In order to measure the shadowing effect, the region around the Moon centre is divided in concentric rings with increasing radius. The event density of each ring is computed as the number of events detected in that sector over the surface of the ring. Event tracks reconstructed as down-going and detected when the Moon is above the Horizon are selected. A test statistic function $\lambda$ is defined to evaluate the statistical significance of the Moon shadow effect:

$$
\lambda=\chi_{H_{1}}^{2}-\chi_{H_{0}}^{2} \text { with } \chi_{H}^{2}=2 \sum_{i=1}^{N_{b i n}}\left[N_{i, H}-n_{i}+n_{i} \ln \frac{n_{i}}{N_{i, H}}\right],
$$

where $n_{i}$ stands for the measurement in the $i$-th bin to be compared with the expectations $N_{i, H}$ under the $H_{0}$ and $H_{1}$ hypotheses.

Using the two Monte Carlo sets mentioned above, $10^{6}$ pseudo-experiments were generated in order to obtain the distribution of the variable $\lambda$ in the case of Moon shadow effect or vice versa (Figure 1).

The dashed area below the dotted curve represents the fraction of pseudo-experiments where the Moon shadow hypothesis is correctly identified; the filled-coloured area below the smooth curve corresponds to a $p$-value equal to $3.6 \times 10^{-4}$, or equivalently $3.4 \sigma$. This is the expected median significance of the Moon shadow effect with the optimized selection criteria.

\section{Results}

The measured muon event density as a function of the angular distance $\delta$ from the Moon is reported below for the data sample of the years 2007-2016, selecting $9.6 \cdot 10^{5}$ events out of the pre-cuts collection about three times larger. (Figure 2). Assuming a Gaussian shape for the detector point spread function, it is possible to evaluate the detector angular resolution fitting the event density with the formula:

$$
\frac{d n}{d \delta^{2}}=k\left(1-\frac{R_{M o o n}^{2}}{2 \sigma_{r e s}^{2}} e^{-\frac{\delta^{2}}{2 \sigma_{r e s}^{2}}}\right)
$$




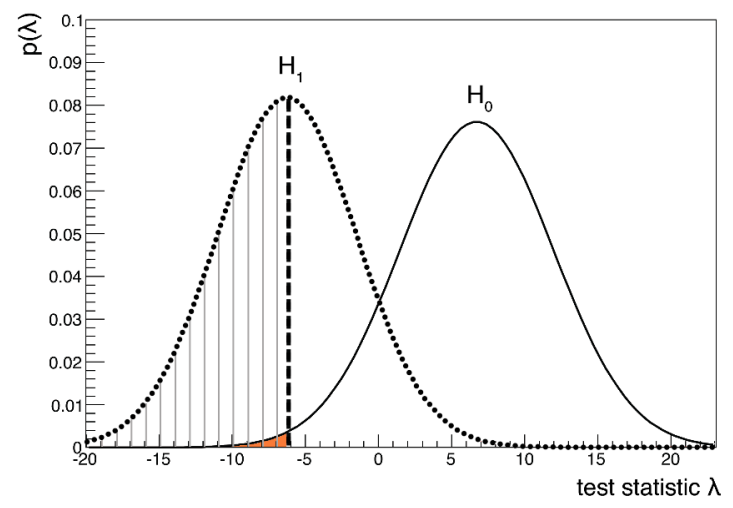

Figure 1. The test statistics $\lambda$ distribution for the "Moon shadow" hypothesis $H_{1}$ (dotted curve) and the "no Moon shadow" hypothesis $H_{0}$ (smooth curve). The dashed area corresponds to the $50 \%$ of the pseudo-experiments where the Moon shadow hypothesis is correctly identified. The shaded area quantifies the expected median significance (here $3.4 \sigma$ ) to observe the Moon shadow.

where $\delta$ is the angular distance from the Moon centre. The two free parameters are $k$, the average muon event density in the off-source region, and $\sigma_{\text {res }}$, the angular resolution. The Moon radius $R_{\text {Moon }}$ is fixed to $0.26^{\circ}$.

The angular resolution for downward-going atmospheric muons resulting from the fit is $\sigma_{\text {res }}=$ $0.73^{\circ} \pm 0.14^{\circ}$. The significance of the shadowing is evaluated using a $\chi^{2}$ test comparing the measured event density with the flat distribution $\frac{d n}{d \delta^{2}}=k$. Such $\chi^{2}$ test leads to a significance of the Moon shadow effect of $3.3 \sigma$.

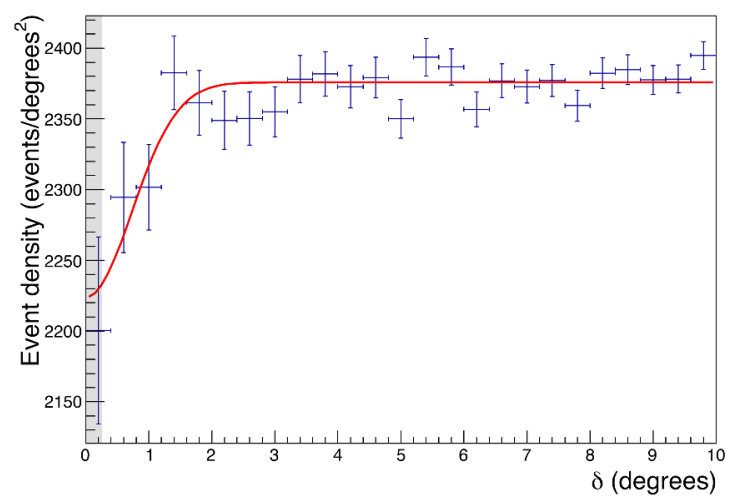

Figure 2. Measured muon event density as a function of the angular distance $\delta$ from the Moon. The smooth line is the best fit according to equation (2); the shaded area corresponds to the apparent radius of the Moon $\left(0.26^{\circ}\right)$. 
The procedure for evaluating the pointing accuracy of the Moon shadow is based on determining the statistical significance of the selected data set under the assumption of the Moon in a given direction. In this study the field of view is limited in both $\mathrm{x}$ and $\mathrm{y}$ within the range $\left[-10^{\circ}, 10^{\circ}\right]$, and it is subdivided in a grid of $0.2^{\circ} \times 0.2^{\circ}$ squared bins. The used test statistic is again $\lambda$ as reported in equation (1), but now the sum is evaluated on all $100 \times 100$ square bins. For each bin in the field of view, the value of the test statistic $\lambda$ is minimised finding the best fit of a bi-dimensional Gaussian point spread function (Figure 3-left) [4]. The smallest value of $\lambda$ is found equal to -17.05 in the bin of coordinates $\left(0.5^{\circ}, 0.1^{\circ}\right)$, this value corresponds to a significance of $3.5 \sigma$ of Moon shadow effect. The interval corresponding to a desired confidence level $(C L)$ (Figure 3-right) can be directly obtained from Figure 3-left accoding to [5].
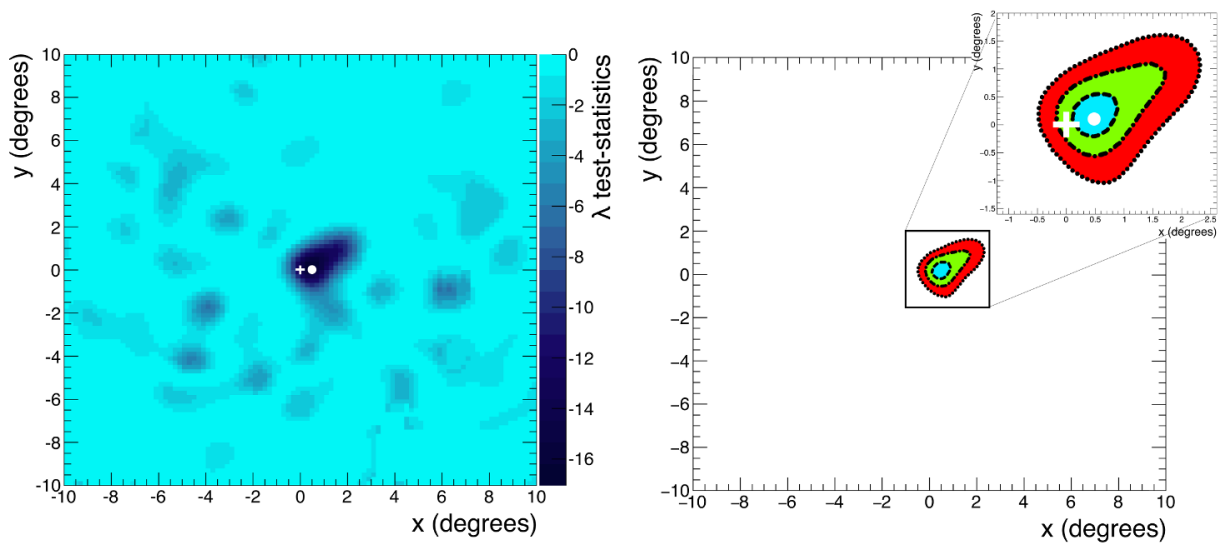

Figure 3. Left: measured distribution of the test statistic $\lambda$ from equation (1) in the field of view around the Moon nominal position $O \equiv\left(0^{\circ}, 0^{\circ}\right)$, indicated by a white cross; the white dot refers to the coordinates $\left(0.5^{\circ}, 0.1^{\circ}\right)$ where the test statistics reaches the minimum. Right: contour plots corresponding to different confidence levels (cyan/dashed: 68.27\%; green/dot-dashed: 95.45\%; red/dotted: 99.73\%); in the zoom the dot represents the position in the FoV where $\lambda$ is minimum, the cross indicates the nominal position of the Moon.

\section{Conclusions}

The pointing performance of the ANTARES telescope has been estimated using the Moon shadow effect. The analysis of the ANTARES data sample of the years 2007-2016 allowed the identification of the Moon shadow effect with $3.5 \sigma$ significance and the detector angular resolution for downwardgoing atmospheric muons of $0.73^{\circ} \pm 0.14^{\circ}$. The pointing accuracy of the detector is consistent with the expectations.

\section{References}

[1] M. Ageron et al. (ANTARES), Nucl. Instrum. Meth. A656, 11 (2011), 1104. 1607

[2] G. Carminati, A. Margiotta, M. Spurio, Comput. Phys. Commun. 179, 915 (2008), 0802 . 0562

[3] S. Adrian-Martinez et al. (ANTARES), JCAP 1303, 006 (2013), 1302.6750

[4] A. Albert et al., The European Physical Journal C 78, 1006 (2018)

[5] G. Cowan, Statistical Data Analysis (Clarendon (Oxford), Oxford, 1998 (ISBN: 0-19-850156-0)) 